\title{
I. Kolár̆
}

Nagoya Math. J.

Vol. 158 (2000), 99-106

\section{AFFINE STRUCTURE ON WEIL BUNDLES}

\author{
IVAN KOLÁŘ
}

\begin{abstract}
For every $r$-th order Weil functor $T^{A}$, we introduce the underlying $k$ th order Weil functors $T^{A_{k}}, k=1, \ldots, r-1$. We deduce that $T^{A} M \rightarrow T^{A_{r-1}} M$ is an affine bundle for every manifold $M$. Generalizing the classical concept of contact element by C. Ehresmann, we define the bundle $\kappa T^{A} M$ of contact elements of type $A$ on $M$ and we describe some affine properties of this bundle.
\end{abstract}

The theory of Weil bundles, [7], is a powerful tool for many general problems in differential geometry. In the seventies, this was testified by Morimoto, [5], and the further development up to the beginning of the nineties is reflected in the monograph [4]. The best known example of a Weil bundle is the bundle $T_{n}^{r} M$ of $n$-dimensional velocities of order $r$ on a manifold $M$. In this case, there are classical proofs of the fact that $T_{n}^{r} M \rightarrow T_{n}^{r-1} M$ is an affine bundle, see e.g. [4, p. 122]. However, a general advantage of the Weil bundle technique is that it often enables us to replace extended calculations by much more concentrated algebraic expressions. From this point of view, the affine bundle structure on $T_{n}^{r} M$ has been studied algebraically in a recent paper by F. J. Muriel, J. Muñoz and J. Rodriguez, [6].

At the beginning of the present paper we point out that every Weil functor $T^{A}$ of order $r$ induces the underlying lower order Weil functors $T^{A_{k}}$, $k=1, \ldots, r-1$. Then we deduce that $T^{A} M \rightarrow T^{A_{r-1}} M$ is an affine bundle. In particular, this general result covers not only the velocities bundles, but also the $r$-th iterated tangent bundle, which was studied systematically by J. E. White, [8]. Next we introduce the contact elements of type $A$ for every Weil algebra $A$, which are equivalent to the $A$-jets by Muriel, Muñoz and Rodriguez, [6]. Using our general results on $T^{A} M$, we describe some affine properties of $\kappa T^{A} M$.

Received January 7, 1999.

1991 Mathematics Subject Classification: 58A20.

This work was accomplished during the author's stay at the Max-Plank Institute for Mathematics in the Sciences, Leipzig. The author was also supported by a grant of the GAČR 201/96/0079. 
All manifolds and maps are assumed to be infinitely differentiable. Unless otherwise specified, we use the terminology and notation from [4]. The author acknowledges R. Alonso, J. Muñoz, J. Muriel and J. Rodriguez for several useful discussions on the subject of this paper.

1. Let $A=\mathbb{R} \times N$ be a Weil algebra, where $N$ is the ideal of all nilpotent elements, [7]. We say that $A$ is of order $r$, if $N^{r+1}=0$ and $N^{r} \neq 0$. We assume $r \geq 2$ in the sequel. The order of $A$ coincides with the order of the Weil functor $T^{A}$ determined by $A$, [4]. We recall that every element $X \in T_{x_{0}}^{A} M$ can be interpreted as an algebra homomorphism of the algebra $C^{\infty} M$ of smooth functions on a manifold $M$ into $A$ of the form

$$
X f=f\left(x_{0}\right)+\widetilde{X} f, \quad \widetilde{X} f \in N, f \in C^{\infty} M
$$

Definition 1. The factor algebra $A_{k}=A / N^{k+1}$ is called the underlying algebra of order $k$. The Weil functor $T^{A_{k}}$ is said to be the underlying $k$-th order functor of $T^{A}$.

So $\pi_{k}: T^{A} M \rightarrow T^{A_{k}} M$ is a surjective submersion. Write

$$
V=N / N^{2}
$$

which is a vector space. Hence $A_{1}=\mathbb{R} \times V$ with zero multiplication in $V$.

Let $B=\mathbb{R} \times P$ be another Weil algebra.

LEMma 1. For every algebra homomorphism $f: A \rightarrow B$, we have $f\left(N^{k}\right) \subset P^{k}, k=1, \ldots, r$.

Proof. For $k=1$, the nilpotency implies $f(N) \subset P$. Next we proceed by iteration.

Proposition 1. Every homomorphism $A \rightarrow B$ factorizes through an underlying homomorphism $f_{k}: A_{k} \rightarrow B_{k}$.

Proof. By Lemma 1, $f$ maps $N^{k+1}$ into $P^{k+1}$. This implies the existence of $f_{k}$.

Taking into account the classical bijection between the homomorphisms of Weil algebras and the natural transformations of the corresponding Weil functors, we obtain 
COROLlaRY 1. Every natural transformation $\tau: T^{A} \rightarrow T^{B}$ factorizes through a natural transformation $\tau_{k}: T^{A_{k}} \rightarrow T^{B_{k}}$.

2. Clearly, $N^{r}$ is a vector space. For every $Z \in T_{x_{0}} M \otimes N^{r}=\operatorname{Lin}\left(T_{x_{0}}^{*} M, N^{r}\right)$ and every $Q \in T_{x_{0}}^{*} M$, we have $Z(Q) \in N^{r}$. We denote by $T_{x_{0}}^{*} f$ the differential of $f \in C^{\infty} M$ at $x_{0} \in M$.

Lemma 2. For every $X \in T_{x_{0}}^{A} M$ and $Z \in T_{x_{0}} M \otimes N^{r}$,

$$
(X+Z) f:=f\left(x_{0}\right)+\widetilde{X} f+Z\left(T_{x_{0}}^{*} f\right), \quad f \in C^{\infty} M
$$

is also an algebra homomorphism.

Proof. For another $g \in C^{\infty} M$, we have

$$
\begin{aligned}
& \left(f\left(x_{0}\right)+\tilde{X} f+Z\left(T_{x_{0}}^{*} f\right)\right)\left(g\left(x_{0}\right)+\widetilde{X} g+Z\left(T_{x_{0}}^{*} g\right)\right) \\
& \quad=\left(f\left(x_{0}\right)+\widetilde{X} f\right)\left(g\left(x_{0}\right)+\widetilde{X} g\right)+Z\left(f\left(x_{0}\right) T_{x_{0}}^{*} g+g\left(x_{0}\right) T_{x_{0}}^{*} f\right) \\
& \quad=(X+Z)(f g)
\end{aligned}
$$

as the other three terms vanish by virtue of $N N^{r}=0$.

Clearly, $X$ and $X+Z$ satisfy $\pi_{r-1}(X)=\pi_{r-1}(X+Z)$. Conversely, let $X, Y \in T_{x_{0}}^{A} M$ satisfy $\pi_{r-1}(X)=\pi_{r-1}(Y)$. Then

$$
Y f=f\left(x_{0}\right)+\widetilde{X} f+D f \quad \text { with } D f \in N^{r} .
$$

Since $X$ and $Y$ are algebra homomorphisms, we have

$$
\begin{aligned}
D(f g) & =\left(f\left(x_{0}\right)+\widetilde{X} f+D f\right)\left(g\left(x_{0}\right)+\widetilde{X} g+D g\right)-X(f g) \\
& =f\left(x_{0}\right) D g+g\left(x_{0}\right) D f
\end{aligned}
$$

by virtue of $N N^{r}=0$. Hence $D$ in an $N^{r}$-valued derivation in $C^{\infty} M$ at $x_{0}$. In the same way as in the proof in 1.5 of [4], we deduce $D \in T_{x_{0}} M \otimes N^{r}$. Thus, we have proved

Proposition 2. $\pi_{r-1}: T^{A} M \rightarrow T^{A_{r-1}} M$ is an affine bundle, whose associated vector bundle is the pullback of $T M \otimes N^{r}$ over $T^{A_{r-1}} M$. 
ExAmples. (i) The Weil algebra of the functor $T_{n}^{r}$ of the $(n, r)$-velocities is

$$
\mathbb{D}_{n}^{r}=\mathbb{R}\left[x_{1}, \ldots, x_{n}\right] /\left\langle x_{1}, \ldots, x_{n}\right\rangle^{r+1},
$$

so that $N^{r}=S^{r} \mathbb{R}^{n *}$. In this case we obtain the classical result mentioned in the introduction.

(ii) The Weil algebra of the $r$-th iterated tangent functor $T^{r}$ is $A=$ $\mathbb{D} \otimes \cdots \otimes \mathbb{D}, \mathbb{D}=\mathbb{D}_{1}^{1}$. One finds easily $N^{r}=\mathbb{R}^{*}$. The underlying bundle of order $r-1$ is the so-called boundary of $T^{r} M=T \cdots T M$, which was studied in [8]. In this case we have rededuced that $T^{r} M$ is an affine bundle over the boundary, whose associated vector bundle is the pullback of TM over the boundary. (The case $r=2$ is well known.)

(iii) Consider the iterated velocities functor $T_{p}^{s} T_{n}^{r}$ of the order $r+s$, whose Weil algebra is $\mathbb{D}_{n}^{r} \otimes \mathbb{D}_{p}^{s}$. One finds easily that the underlying bundle of the order $r+s-1$ is the fiber product

$$
T_{p}^{s-1} T_{n}^{r} M \times_{T_{p}^{s-1} T_{n}^{r-1} M} T_{p}^{s} T_{n}^{r-1} M
$$

and the vector bundle in question is

$$
T M \otimes\left(S^{r} \mathbb{R}^{n *}\right) \otimes\left(S^{s} \mathbb{R}^{p *}\right)
$$

Consider a smooth map $\varphi: M \rightarrow Q$.

Proposition 3. $T^{A} \varphi: T^{A} M \rightarrow T^{A} Q$ is an affine bundle morphism over $T^{A_{r-1}} \varphi: T^{A_{r-1}} M \rightarrow T^{A_{r-1}} Q$, whose associated vector bundle morphism is the pullback of $T \varphi \otimes \mathrm{id}_{N^{r}}$.

Proof. Let $D$ be as in (2). For every $f \in C^{\infty} Q$, write $\left(\varphi_{*} D\right)(f)=$ $D(f \circ \varphi)$. Since $D$ is an $N^{r}$-valued derivation at $x_{0} \in M, \varphi_{*} D$ is an $N^{r}$ valued derivation at $\varphi\left(x_{0}\right) \in Q$.

3. The group Aut $A$ of all algebra automorphisms of $A$ is a Lie group, [4]. By Proposition 1, we have a canonical group homomorphism Aut $A \rightarrow$ Aut $A_{k}$ (which is not surjective in general).

Proposition 4. The kernel $K$ of the canonical homomorphism Aut $A$ $\rightarrow$ Aut $A_{r-1}$ is the Abelian group $V^{*} \otimes N^{r}$. 
Proof. Let $p: N \rightarrow V=N / N^{2}$ be the factor projection. For $Z \in$ $V^{*} \otimes N^{r}=\operatorname{Lin}\left(V, N^{r}\right)$, we define $\zeta: A \rightarrow A$ by

$$
\zeta(a+n)=a+n+Z(p(n)), \quad a \in \mathbb{R}, n \in N .
$$

This is an algebra homomorphism, for

$$
\begin{aligned}
\zeta\left(\left(a_{1}+n_{1}\right)\left(a_{2}+n_{2}\right)\right) & =\left(a_{1}+n_{1}\right)\left(a_{2}+n_{2}\right)+Z\left(a_{2} p\left(n_{1}\right)+a_{1} p\left(n_{2}\right)\right) \\
& =\left(a_{1}+n_{1}+Z\left(p\left(n_{1}\right)\right)\right)\left(a_{2}+n_{2}+Z\left(p\left(n_{2}\right)\right)\right) .
\end{aligned}
$$

For another $S \in V^{*} \otimes N^{r}$, we have

$$
\sigma(\zeta(a+n))=\sigma(a+n+Z(p(n)))=a+n+Z(p(n))+S(p(n)) .
$$

Hence this is a group homomorphism of an Abelian group.

Conversely, let $\zeta \in$ Aut $A$ be over $\operatorname{id}_{A_{r-1}}$. We define

$$
D(n)=\zeta(n)-n \in N^{r}, \quad n \in N .
$$

Hence $D: N \rightarrow N^{r}$ is a linear map. By $N N^{r}=0$, every $v, w \in N$ satisfy $v(\zeta(w)-w)=0$, i.e., $v \zeta(w)=v w$. So

$$
0=D(v) D(w)=\zeta(v) \zeta(w)-v w-v w+v w=D(v w) .
$$

This implies $D(n+v w)=D(n)$, so that $D$ factorizes through a linear map $V \rightarrow N^{r}$.

Since every algebra homomorphism $Z: A \rightarrow A$ induces a natural transformation (denoted by the same symbol) $Z: T^{A} \rightarrow T^{A}$, we have a canonical action of Aut $A$ on $T^{A} M,(Z, X) \mapsto Z(X), X \in T^{A} M$. We are going to deduce an explicit formula for the restriction of this action to $V^{*} \otimes N \subset$ Aut $A$. We shall need a lemma.

Lemma 3. We have $T^{A_{1}} M=T M \otimes V$.

Proof. Since $A_{1}=\mathbb{R} \times V$ with zero multiplication in $V$, the $\widetilde{X}$ induced by (1) from $X \in T_{x_{0}}^{A_{1}} M$ is a $V$-valued derivation in $C^{\infty} M$ at $x_{0}$, i.e., an element of $T_{x_{0}} M \otimes V$, and vice versa. 
Let $Z \in V^{*} \otimes N^{r}$. In the notation of (3), we have

$$
\zeta(X f)=f\left(x_{0}\right)+\widetilde{X} f+Z(p(\widetilde{X} f))
$$

By the definition of $\pi_{1}$ and by Lemma 3 , the formula $\widetilde{\pi_{1}(X)} f=p(\widetilde{X} f)$ defines a map

$$
\widetilde{\pi_{1} X}: T_{x_{0}}^{*} M \longrightarrow V \text {. }
$$

Since $Z: V \rightarrow N^{r}$, we have $Z \circ \widetilde{\pi_{1}(X)} \in T_{x_{0}} M \otimes N^{r}$. Thus, (4) can be rewritten as

$$
Z(X)=X+Z \circ \widetilde{\pi_{1}(X)}
$$

where $Z \circ \widetilde{\pi_{1}(X)}$ is an element of the associated vector bundle of $T^{A} M$.

4. Our Definition 3 below is equivalent to 1.1 .3 from [6], but we use the approach developed in [4]. We recall that an element $X \in T^{A} M$ is said to be an $A$-velocity on $M$.

Definition 2. An $A_{1}$-velocity $X \in T_{x_{0}}^{A_{1}} M$ is said to be regular, if $\widetilde{X}$ is injective as a map $V^{*} \rightarrow T_{x_{0}} M$. An $A$-velocity $X$ is called regular, if $\pi_{1}(X)$ is regular.

We denote by $\operatorname{reg} T^{A} M$ the bundle of all regular $A$-velocities on $M$. If $\varphi: M \rightarrow Q$ is an immersion, then $T^{A} \varphi$ transforms $\operatorname{reg} T^{A} M$ into $\operatorname{reg} T^{A} Q$. The restricted and corestricted map will be denoted by $\operatorname{reg} T^{A} \varphi$. Hence $\operatorname{reg} T^{A}$ is a bundle functor on the category $\mathcal{I} m m$ of all immersions.

The jet group $G_{n}^{r}$ of order $r$ in dimension $n$ acts on $\operatorname{reg} T_{n}^{r} M$ by the jet composition. The equivalence classes are called regular contact elements by C. Ehresmann, [2], or contact element in [4]. The manifold structure on the bundle of all contact $(n, r)$-elements

$$
K_{n}^{r} M=\operatorname{reg} T_{n}^{r} M / G_{n}^{r}
$$

is described in [4, p. 124], see also [3]. For every immersion $\varphi: M \rightarrow Q$, we have the induced map $K_{n}^{r} \varphi: K_{n}^{r} M \rightarrow K_{n}^{r} Q$.

We shall need a vector bundle $\varrho_{n} M$ over $K_{n}^{1} M$, whose fibers are defined as follows. Every $X \in\left(K_{n}^{1} M\right)_{x_{0}}$ is identified with an $n$-dimensional linear subspace $\sigma(X) \subset T_{x_{0}} M$ and the fiber $\left(\varrho_{n} M\right)_{X}$ is the factor space $T_{x_{0}} M / \sigma(X)$. Obviously, every immersion $\varphi: M \rightarrow Q$ induces canonically a $\operatorname{map} \varrho_{n} \varphi: \varrho_{n} M \rightarrow \varrho_{n} Q$. 
5. The group $G_{n}^{r}$ coincides with Aut $\mathbb{D}_{n}^{r}$ and its action on $T_{n}^{r} M$ is that of item 3, [4]. So we introduce the following generalization of the notion of contact element, which is equivalent to the concept of $A$-jet from [6].

Definition 3. The contact element determined by $X \in \operatorname{reg} T^{A} M$ is the equivalence class

$$
\kappa X=(\text { Aut } A) X=\{Z(X) ; Z \in \text { Aut } A\} .
$$

We denote by $\kappa T^{A} M$ the set of all contact $A$-elements on $M$. R. Alonso deduced that $\kappa T^{A} M$ is a smooth manifold and $\operatorname{reg} T^{A} M \rightarrow \kappa T^{A} M$ is a smooth principal fiber bundle with structrure group Aut $A$, [1]. Every immersion $\varphi: M \rightarrow Q$ induces a map $\kappa T^{A} \varphi: \kappa T^{A} M \rightarrow \kappa T^{A} Q$. The canonical projection $\mu_{k}: \kappa T^{A} M \rightarrow \kappa T^{A_{k}} M$ is defined by $\mu_{k}(($ Aut $A) X)=$ $\left(\right.$ Aut $\left.A_{k}\right) \pi_{k}(X)$. For $r=1, \kappa T^{A_{1}} M=K_{n}^{1} M, n=\operatorname{dim} V$.

Proposition 5. Let $X, Y \in \operatorname{reg} T_{x_{0}}^{A} M$ have the same projection into $T_{x_{0}-1}^{A_{r-1}} M$. Then there exists $Z \in V^{*} \otimes N$ satisfying $Z(X)=Y$ if and only if

$$
Y-X \in \sigma\left(\pi_{1}(X)\right) \otimes N^{r} \subset T_{x_{0}} M \otimes N^{r}
$$

Proof. By (5), we have $Z(X)-X=Z \circ \widetilde{\pi_{1} X} \in \sigma\left(\pi_{1}(X)\right) \otimes N^{r}$. Conversely, consider the injection $i: \sigma\left(\pi_{1}(X)\right) \rightarrow T_{x_{0}} M$. Then (6) means that $Y-X: T_{x_{0}}^{*} M \rightarrow N^{r}$ is of the form $W \circ i^{*}$ with $W: \sigma\left(\pi_{1}(X)\right)^{*} \rightarrow N^{r}$. Since $\pi_{1}(X)$ is regular, $\widetilde{\pi_{1}(X)}: T_{x_{0}}^{*} M \rightarrow V$ is of the form $\mu \circ i^{*}$, where $\mu: \sigma\left(\pi_{1}(X)\right)^{*} \rightarrow V$ is invertible. Define $Z=W \circ \mu^{-1}: V \rightarrow N^{r}$. Then $Z(X)=X+W \circ \mu^{-1} \circ \widetilde{\pi_{1} X}=X+W \circ i^{*}=X+Y-X=Y$.

Thus, if we fix an element $S \in \operatorname{reg} T^{A_{r-1}} M$, we have defined an affine space structure of the subset $\widetilde{S} \subset\left(\kappa T^{A} M\right)_{\kappa(S)}$ of the form $\widetilde{S}=\{\kappa(X), X \in$ $\left.\operatorname{reg} T^{A} M ; \pi_{r-1}(X)=S\right\}$. The associated vector space is $\left(\varrho_{n} M\right)_{\kappa\left(S_{1}\right)} \otimes N^{r}$, where $S_{1}$ is the canonical projection of $S$ into $T^{A_{1}} M$.

The simpliest situation is in the case $\widetilde{S}$ coincides with $\left(\kappa T^{A} M\right)_{\kappa(S)}$ for every $S \in \operatorname{reg} T^{A_{r-1}} M$. Then the above construction endows the fiber of $\kappa T^{A} M$ over each point $X \in \kappa T^{A_{r-1}} M$ with the structure of an affine space. These affine structures on $\left(\kappa T^{A} M\right)_{X}$ are parametrized by the elements $S \in$ $\operatorname{reg} T^{A_{r-1}} M$ satisfying $\kappa(S)=X$. In particular, this is true for the bundle $K_{n}^{r} M$ of the classical contact elements. 


\section{REFERENCES}

[1] R. Alonso, Jet manifold associated to a Weil bundle, to appear in Archivum Math. (Brno).

[2] C. Ehresmann, Introduction à la théorie des structures infinitésimales et des pseudogroupes de Lie, Colloque du C.N.R.S., Strasbourg (1953), 97-110.

[3] D. R. Grigore and D. Krupka, Invariants of velocities and higher order Grassmann bundles, J. Geom. Phys., 24 (1998), 244-264.

[4] I. Koláŕ, P. W. Michor and J. Slovák, Natural Operations in Differential Geometry, Springer-Verlag, 1993.

[5] A. Morimoto, Prolongations of connections to bundles of infinitely near points, J. Diff. Geo., 11 (1976), 479-498.

[6] F. J. Muriel, J. Muñoz and J. Rodriguez, Weil bundles and jet spaces, to appear in Czechoslovak Math. J.

[7] A. Weil, Théorie des points proches sur les variétés différentiables, Colloque de C.N.R.S., Strasbourg (1953), 111-117.

[8] J. E. White, The method of iterated tangents with applications to local Riemannian geometry, Pitman Press, 1982.

Department of Algebra and Geometry

Masaryk University

Janáčkovo nám. $2 a$

662 95 Brno

Czechia

kolar@math.muni.cz 